\title{
Single Patient Classifier Assay, Microsatellite Instability, and Epstein-Barr Virus Status Predict Clinical Outcomes in Stage II/III Gastric Cancer: Results from CLASSIC Trial
}

\author{
Chul Kyu Roh ${ }^{1 *}$, Yoon Young Choi ${ }^{1,2 *}$, Seohee $\mathrm{Choi}^{1}$, Won Jun Seo ${ }^{1}$, Minah Cho ${ }^{1}$, \\ Eunji Jang ${ }^{3}$, Taeil Son ${ }^{1}$, Hyoung-Il Kim ${ }^{1}$, Hyeseon Kim ${ }^{3}$, Woo Jin Hyung ${ }^{1}$, \\ Yong-Min Huh ${ }^{2,3,4,5,6}$, Sung Hoon Noh ${ }^{1,7}$, and Jae-Ho Cheong ${ }^{1,2,5,7}$ \\ ${ }^{1}$ Department of Surgery, Yonsei University Health System, Seoul; \\ ${ }^{2}$ Yonsei Biomedical Research Institute, Yonsei University Health System, Seoul; \\ ${ }^{3}$ MediBio-Informatics Research Center, Novomics Co., Ltd., Seoul; \\ ${ }^{4}$ Department of Radiology, Yonsei University Health System, Seoul; \\ ${ }^{5}$ Department of Biochemistry \& Molecular Biology, Yonsei University Health System, Seoul; \\ ${ }^{6}$ YUHS-KRIBB Medical Convergence Research Institute, Seoul; \\ ${ }^{7}$ Brain Korea 21 PLUS Project for Medical Science, Yonsei University College of Medicine, Seoul, Korea.
}

Purpose: Clinical implications of single patient classifier (SPC) and microsatellite instability (MSI) in stage II/III gastric cancer have been reported. We investigated SPC and the status of MSI and Epstein-Barr virus (EBV) as combinatory biomarkers to predict the prognosis and responsiveness of adjuvant chemotherapy for stage II/III gastric cancer.

Materials and Methods: Tumor specimens and clinical information were collected from patients enrolled in CLASSIC trial, a randomized controlled study of capecitabine plus oxaliplatin-based adjuvant chemotherapy. The results of nine-gene based SPC assay were classified as prognostication (SPC-prognosis) and prediction of chemotherapy benefit (SPC-prediction). Five quasimonomorphic mononucleotide markers were used to assess tumor MSI status. EBV-encoded small RNA in situ hybridization was performed to define EBV status.

Results: There were positive associations among SPC, MSI, and EBV statuses among 586 patients. In multivariate analysis of disease-free survival, SPC-prognosis [hazard ratio (HR): 1.879 (1.101-3.205), 2.399 (1.415-4.067), $p=0.003$ ] and MSI status (HR: 0.363, 95\% confidence interval: 0.161-0.820, $p=0.015)$ were independent prognostic factors along with age, Lauren classification, TNM stage, and chemotherapy. Patient survival of SPC-prognosis was well stratified regardless of EBV status and in microsatellite stable (MSS) group, but not in MSI-high group. Significant survival benefit from adjuvant chemotherapy was observed by SPC-Prediction in MSS and EBV-negative gastric cancer.

Conclusion: SPC, MSI, and EBV statuses could be used in combination to predict the prognosis and responsiveness of adjuvant chemotherapy for stage II/III gastric cancer.

Key Words: Gastric cancer, microsatellite instability, single patient classifier, EBV

Received: December 20, 2018 Revised: December 26, 2018 Accepted: December 27, 2018

Corresponding author: Jae-Ho Cheong, MD, PhD, Department of Surgery, Yonsei University College of Medicine, 50-1 Yonsei-ro, Seodaemun-gu, Seoul 03722, Korea. Tel: 82-2-2228-2094, Fax: 82-2-313-8289, E-mail: JHCHEONG@yuhs.ac

${ }^{*}$ Chul Kyu Roh and Yoon Young Choi contributed equally to this work.

- Jae-Ho Cheong, Yong-Min Huh, and Sung Hoon Noh are the founders and shareholders of Novomics Co., Ltd., and they have applied for a patent.

(c) Copyright: Yonsei University College of Medicine 2019

This is an Open Access article distributed under the terms of the Creative Commons Attribution Non-Commercial License (https://creativecommons.org/licenses/by-nc/4.0) which permits unrestricted non-commercial use, distribution, and reproduction in any medium, provided the original work is properly cited. 


\section{INTRODUCTION}

Surgery and chemotherapy are the main treatment strategies against most solid cancers. Recently developed technologies and updates in the understanding of cancer biology have made it possible to classify cancers according to their molecular characteristics. These achievements have opened the era of precision medicine against cancer, ${ }^{1-3}$ and various biomarkers and algorithms are being used in clinical practice to decide treatment strategies, as they can predict the prognosis and responsiveness to chemotherapy in various cancers. ${ }^{4-6}$

Gastric cancer is one of the most common and lethal malignancies in the world, especially in Korea. Various efforts have been made to understand gastric cancer at molecular level, hoping that the findings may have clinical utility. ${ }^{7-9}$ For example, through integrated analyses using multi-omics platforms, the Cancer Genome Atlas study has identified four molecular subtypes of gastric cancer, including Epstein-Barr virus (EBV), microsatellite instability (MSI), chromosomal instability (CIN), and genomic stable (GS) types, with distinctive biological characteristics. Although a promising utility of MSI in making clinical decisions has been extensively reported, ${ }^{10-14}$ concrete evidence is still needed to confirm clinical utility of other subtypes; currently, no assay is available for applying routine use to identify certain molecular subtypes such as CIN and GS, which prevents the accumulation of standardized evidence. In this regard, we have developed a predictive test on GMP-grade qRT-PCR assay platform for predicting the prognosis and response to adjuvant chemotherapy after D2 gastrectomy for stage II/III gastric cancer: single patient classifier (SPC) ${ }^{15,16}$ Based on gene expression profiling, SPC translates clinically relevant molecular characteristics in gastric cancer (i.e., immune, stem-like, and epithelial type characteristics) into SPC-prognosis (low, intermediate, and high-risk groups) and SPC-prediction subtypes (responder and non-responder groups for adjuvant chemotherapy), serving as an accessible tool for the molecular subtyping of gastric cancer, along with EBV or MSI.

Taken together, we believe that from a practical standpoint, it would be worthwhile to evaluate the combinatory effects of clinically relevant subtypes, such as SPC, MSI, and EBV, in order to make a more precise stratification of patients for their prognosis and predict their responsiveness to adjuvant chemotherapy.

\section{MATERIALS AND METHODS}

\section{Study design and patients}

From a total of 1035 patients enrolled in CLASSIC trial, which is a randomized controlled study of capecitabine plus oxaliplatin-based adjuvant chemotherapy for stage II/III gastric cancer, ${ }^{17,18}$ we reviewed the medical records of 586 patients, for whom it was possible to identify SPC, MSI and EBV statuses. Of these, 282 patients underwent $\mathrm{D} 2$ gastrectomy alone, while 304 patients underwent D2 gastrectomy plus adjuvant chemotherapy. This study was approved by the Institutional Review Board at Severance Hospital, Yonsei University Health System (IRB No: 4-2016-0068, 4-2016-0433).

\section{Definition of SPC status}

We had previously analyzed the expression of four classifier genes (GZMB and WARS representing immune module, $S F R P 4$ representing stem-like module, and CDX1 representing intestinal epithelial module) and five reference genes ( ACTB, ATP5E, HPRT1, GPX1, and UBB) by performing a realtime RT-PCR assay (nProfiler 1 Stomach Cancer Assay, Novomics Co., Ltd., Seoul, Korea) using samples from CLASSIC trial cohort. ${ }^{15}$ Prognostication (SPC-prognosis; low-risk, intermediate-risk or high-risk) and prediction of chemotherapy benefit (SPC-prediction; responder or non-responder) were defined according to the expression results of these nine genes from SPC assay.

\section{Definition of MSI status}

Previously, we had also analyzed MSI status from CLASSIC trial cohort using a panel (MSI Analysis System, Version 1.2; Promega, Madison, WI, USA) of five quasi-monomorphic mononucleotide repeat markers (NR-21, BAT-25, MONO-27, NR-24, and BAT-26) that determined MSI status of the tumor with high accuracy. ${ }^{13}$ MSI-H was defined as instability of two or more of the five markers, whereas MSI-L and microsatellite stable (MSS) were defined as instability of one marker and those without instability. ${ }^{19,20}$

\section{Epstein-Barr virus-encoded small RNA in situ hybridization}

Two core tissue microarrays, with a diameter of $3 \mathrm{~mm}$ for each case, were constructed from formalin-fixed paraffin-embedded tumor blocks. Ventana Benchmark ISH system (Ventana ISH iView kit, Ventana Corporation, Tucson, AZ, USA) was used for Epstein-Barr virus-encoded small RNA in situ hybridization (EBER-ISH). ${ }^{21}$ The presence of EBV in tumor cells in EBER-ISH analysis was considered as a positive result; the absence of EBV was considered an EBV-negative result.

\section{Statistical analysis}

Chi-square test or Fisher's exact test were performed for categorical variables. Continuous variables were analyzed with independent $t$ test. Disease-free survival (DFS) was defined as the time from surgery to recurrence or death, whichever occurred first. Survival data were censored at the last time of follow-up or at seven years for patients without these events. DFS curves were generated using Kaplan-Meier method and log-rank test. Cox proportional hazards regression models were used to obtain hazard ratios (HRs) with $95 \%$ confidence 
interval (CI). The forward likelihood-ratio model was used for selecting inclusion variables in the final multivariable model, using an entry criterion between 0.15 and 0.25 as significance level. Two-sided $p$ values less than 0.05 were considered statistically significant. Data were analyzed using IBM SPSS version 23.0 (IBM Corp., Armonk, NY, USA).

\section{RESULTS}

\section{Baseline characteristics of study cohort}

Table 1 shows clinical information of the study cohort $(n=586)$ of the current study. Between the original CLASSIC cohort $(n=1035)$ and this collected cohort, no significant differences were observed; ${ }^{13,15} 70$ (11.9\%), 278 (47.4\%), and 238 (40.6\%) patients were classified into low-, intermediate-, and high-risk groups, respectively, by SPC-prognosis, and 318 (54.3\%) and $268(45.7 \%)$ of patients were classified as non-responders and

Table 1. Clinical Characteristics of Entire Cohort

\begin{tabular}{|c|c|}
\hline & Entire cohort $(n=586)$ \\
\hline Age (yr) & $56.1 \pm 11.6$ \\
\hline \multicolumn{2}{|l|}{ Sex } \\
\hline Male & $410(70.0)$ \\
\hline Female & $176(30.0)$ \\
\hline \multicolumn{2}{|l|}{ Lauren classification } \\
\hline Intestinal & $215(36.7)$ \\
\hline Non-intestinal & $371(63.3)$ \\
\hline \multicolumn{2}{|l|}{ EBV status } \\
\hline Negative & $541(93.0)$ \\
\hline Positive & $41(7.0)$ \\
\hline \multicolumn{2}{|l|}{ MSI status } \\
\hline MSS/MSI-L & 547 (93.3) \\
\hline MSI-H & $39(6.7)$ \\
\hline \multicolumn{2}{|l|}{ Treatment } \\
\hline Surgery only & $282(48.1)$ \\
\hline Surgery+CTx & $304(51.9)$ \\
\hline \multicolumn{2}{|l|}{ pTNM stage* } \\
\hline$\|$ & $276(47.1)$ \\
\hline III & $310(52.9)$ \\
\hline \multicolumn{2}{|l|}{ SPC-prognosis } \\
\hline Low-risk & $70(11.9)$ \\
\hline Intermediate-risk & $278(47.4)$ \\
\hline High-risk & $238(40.6)$ \\
\hline \multicolumn{2}{|l|}{ SPC-prediction } \\
\hline Non-responder & $318(54.3)$ \\
\hline Responder & $268(45.7)$ \\
\hline
\end{tabular}

EBV, Epstein-Barr virus; MSI, microsatellite instability; MSS, microsatellite stable; MSI-L, microsatellite instability-low; MSI-H, microsatellite instabilityhigh; CTx, adjuvant chemotherapy (capecitabine plus oxaliplatin); SPC, single patient classifier.

Data are expressed as mean \pm standard deviation or as $n(\%)$.

${ }^{*}$ According to American Joint Committee on Cancer 6th edition. responders, respectively, by SPC-prediction. Furthermore, 39 (6.7\%) patients showed MSI-H tumors and 41 patients $(7.0 \%)$ showed EBV-positive tumors. Detailed baseline and clinical demographics by SPC and MSI have been reported in previous studies. ${ }^{13,15}$ With regards to EBV status, no statistically significant distribution was observed between EBV-positive and -negative cases for clinical variables such as age, Lauren classification, pathologic stages, and treatment, but not gender; most cases of EBV-positive gastric cancer were observed in men (38 out of 41 patients, $92.7 \%, p=0.001$; chi-square test) (Table 2).

\section{Associations of MSI and EBV statuses with respect to SPCs}

With regards to SPC-prognosis, both MSI and EBV statuses had a positive association (Table 3). MSI-H and EBV-positive tumors were dominant in low-risk group compared to that in other groups ( $p<0.001$ for both statuses; chi-square test). With regards to SPC-prediction, 27 (69.2\%) of $39 \mathrm{MSI}-\mathrm{H}$ patients and $38(92.7 \%)$ of 41 EBV-positive patients were enriched in non-responder group ( $p=0.066$ and $<0.001$, respectively). Aside from SPC, MSI and EBV statuses were almost mutually exclusive; only two patients had both MSI-H and EBV-positive gastric cancer $(p>0.999)$.

Table 2. Clinical Characteristics of Patients according to EBV Status

\begin{tabular}{|c|c|c|c|}
\hline & & V status & \\
\hline & $\begin{array}{c}\text { Negative } \\
(n=541)\end{array}$ & $\begin{array}{c}\text { Positive } \\
\text { (n=41) }\end{array}$ & $p$ value \\
\hline Age (yr) & $56.2 \pm 11.6$ & $55.9 \pm 11.5$ & 0.885 \\
\hline Sex & & & 0.001 \\
\hline Male & $371(68.6)$ & 38 (92.7) & \\
\hline Female & $170(31.4)$ & $3(7.3)$ & \\
\hline Lauren classification & & & 0.486 \\
\hline Intestinal & $201(37.2)$ & 13 (31.7) & \\
\hline Non-intestinal & 340 (62.8) & $28(68.3)$ & \\
\hline pTNM stage* & & & 0.274 \\
\hline$\|$ & $259(47.9)$ & $16(39.0)$ & \\
\hline III & $282(52.1)$ & $25(61.0)$ & \\
\hline pT classification* & & & 0.408 \\
\hline $\mathrm{pT} 1 / \mathrm{T} 2$ & $300(55.5)$ & $20(48.8)$ & \\
\hline $\mathrm{pT} 3 / \mathrm{T4}$ & $241(44.5)$ & $21(51.2)$ & \\
\hline pN classification* & & & 0.379 \\
\hline pNO & $48(8.9)$ & $2(4.9)$ & \\
\hline pN1/N2/N3 & $493(91.1)$ & $39(95.1)$ & \\
\hline Treatment & & & 0.663 \\
\hline Surgery only & $258(47.7)$ & $21(51.2)$ & \\
\hline Surgery+CTx & 283 (52.3) & $20(48.8)$ & \\
\hline
\end{tabular}

EBV, Epstein-Barr Virus; CTx, adjuvant chemotherapy (capecitabine plus oxaliplatin).

Data are expressed as mean \pm standard deviation or as $\mathrm{n}(\%)$, unless otherwise specified.

${ }^{*}$ According to American Joint Committee on Cancer 6th edition. 


\section{Prognosis according to markers}

In the study cohort, both SPC and MSI statuses were prognostic factors, but EBV was not ( $p=0.001$ in SPC-prognosis, $p=0.003$ in MSI-H vs. MSS/MSI-L, $p=0.547$ in EBV positive vs. EBV negative; log-rank test).

We first examined the combinatory effects of SPC and MSI statuses in prognostication. In Fig. 1A, different prognoses of low-, intermediate-, and high-risk groups obtained from SPCprognosis were well-preserved in MSS/MSI-L patients [HR: 1.787 (1.000-3.191) in intermediate-risk, 2.510 (1.411-4.463) in high-risk group, log-rank $p=0.001]$, but not in MSI-H patients ( $\log$-rank $p=0.316)$, indicating that SPC and MSI statuses were independent of one another in prognostication. A multivariate analysis using Cox proportional hazards regres-

Table 3. Associations between SPC, MSI, and EBV Status

\begin{tabular}{|c|c|c|c|c|c|c|c|c|c|c|}
\hline & \multicolumn{4}{|c|}{ SPC-prognosis } & \multicolumn{3}{|c|}{ SPC-prediction } & \multicolumn{3}{|c|}{ EBV } \\
\hline & Low-risk & Intermediate-risk & High-risk & $p$ value & Non-responder & Responder & $p$ value & Negative & Positive & $p$ value \\
\hline MSI status & & & & $<0.001$ & & & 0.066 & & & $>0.999$ \\
\hline MSS/MSI-L & $57(81.4)$ & 261 (93.9) & $229(96.2)$ & & $291(91.5)$ & $256(95.5)$ & & $504(93.2)$ & 39 (95.1) & \\
\hline MSI-H & $13(18.6)$ & $17(6.1)$ & $9(3.8)$ & & $27(8.5)$ & $12(4.5)$ & & $37(6.8)$ & $2(4.9)$ & \\
\hline EBV & & & & $<0.001$ & & & $<0.001$ & & & \\
\hline Negative & $47(68.1)$ & $262(94.6)$ & 232 (98.3) & & $276(87.9)$ & $265(98.9)$ & & & & \\
\hline Positive & 22 (31.9) & $15(5.4)$ & $4(1.7)$ & & $38(12.1)$ & $3(7.3)$ & & & & \\
\hline
\end{tabular}

SPC, single patient classifier; MSI, microsatellite instability; EBV, Epstein-Barr virus; MSS, microsatellite stable; MSI-L, microsatellite instability-low; MSI-H, microsatellite instability-high.

Data are expressed as $n(\%)$.
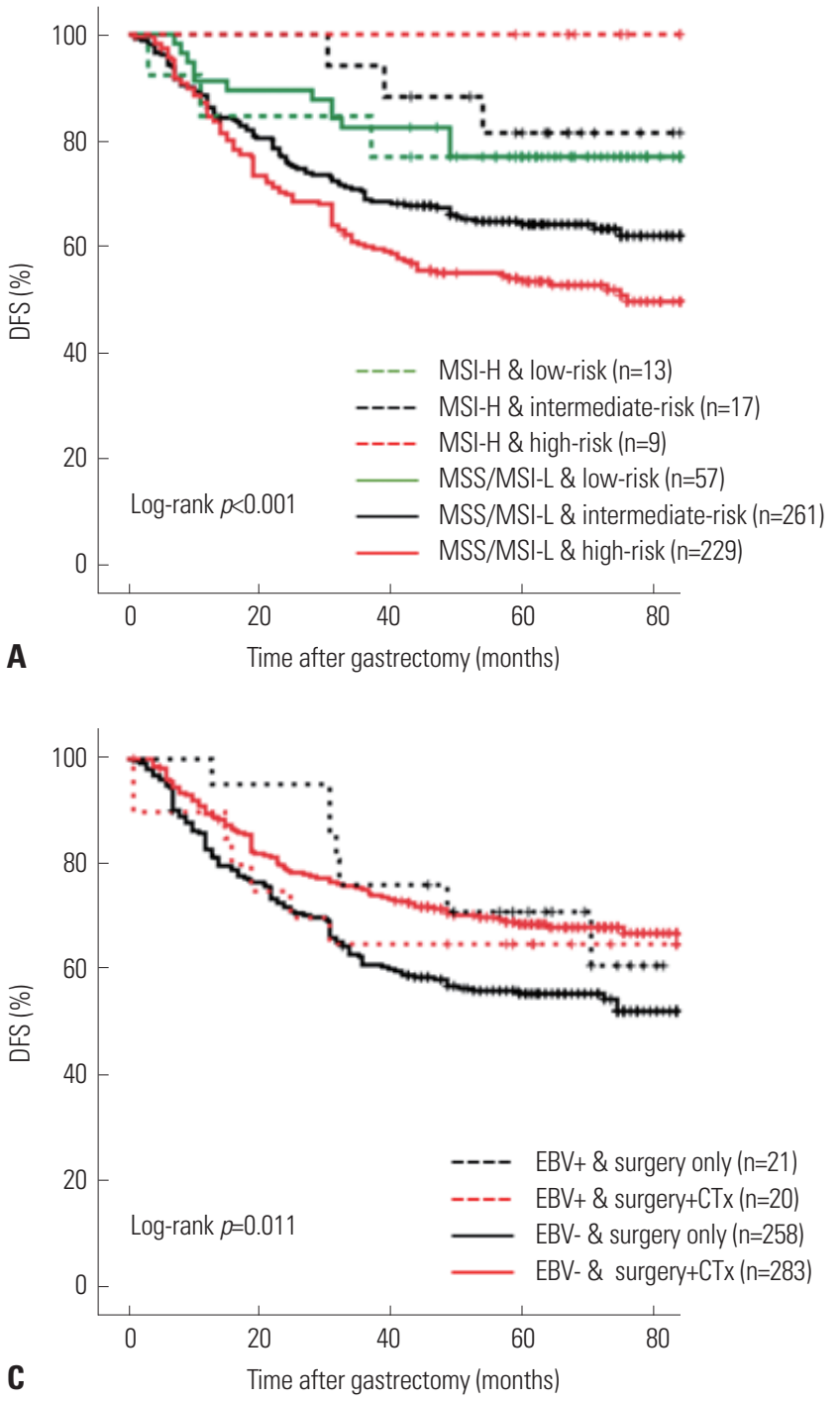

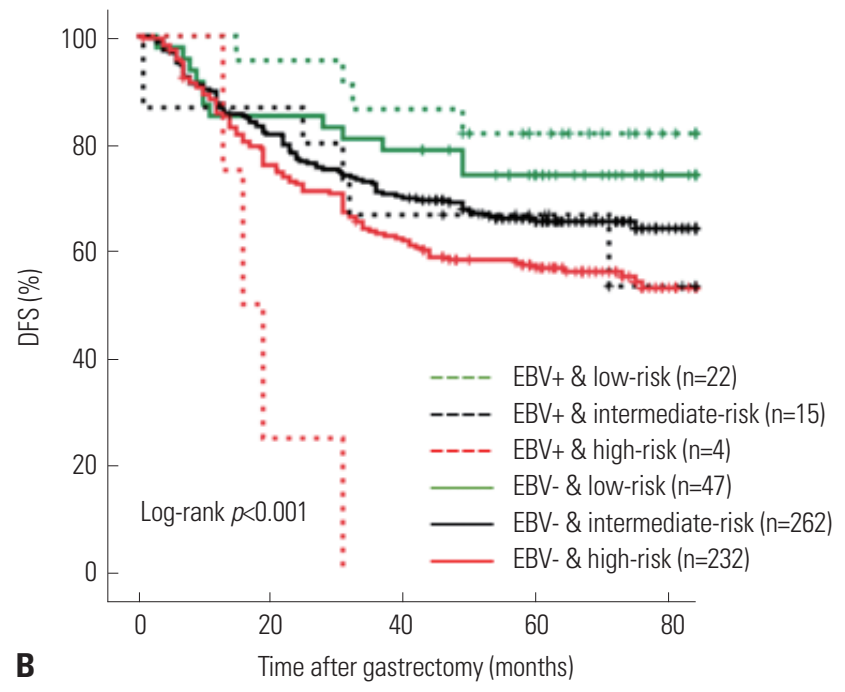

Fig. 1. Kaplan-Meier and log-rank comparisons of DFS in patients with stage II/III gastric cancer according to (A) status of MSI and SPC-prognosis, (B) status of EBV and SPC-prognosis, (C) EBV status and treatment. DFS, disease-free survival; MSI, microsatellite instability; SPC, single patient classifier; MSS, microsatellite stable; MSI-L, microsatellite instability-low; MSI-H, microsatellite instability-high; EBV, Epstein-Barr virus; CTx, adjuvant chemotherapy (capecitabine plus oxaliplatin). 
sion models showed that both MSI status (HR: 0.363, 95\% CI: 0.161-0.820, $p=0.015$ ) and SPC-prognosis [HR: 1.879 (1.1013.205) in intermediate-risk, 2.399 (1.415-4.067) in high-risk group, $p=0.003$ ] were independently significant prognostic factors for DFS, in addition to age, chemotherapy, pTNM stage, and Lauren classification (Table 4).

In contrast, a combination of SPC and EBV statuses revealed a distinct trend whereby the survival by SPC-prognosis was well-stratified regardless of EBV status (Fig. 1B). Intriguingly, in EBV-positive patients, the difference in DFS between SPC-prognosis groups was highly significant [HR: 2.608 (0.735-9.254) in intermediate-risk, 17.656 (3.839-81.200) in high-risk group, log-rank $p<0.001]$, necessitating further investigation. In a multivariate analysis, EBV status was not found to be a significant prognostic factor for DFS [HR: 0.735 (0.425-1.268), $p=0.268]$, in addition to age, sex, chemotherapy, and pTNM stage.

\section{Responsiveness to adjuvant chemotherapy according to markers}

Statistically significant survival benefit from adjuvant chemotherapy was observed in responder group for SPC-prediction, MSS/MSI-L groups, and EBV-negative groups ( $p=0.001,0.002$, and 0.001 , respectively; log-rank test), but not in non-responder group for SPC-prediction, MSI-H groups, and EBV-positive groups ( $p=0.243,0.931$, and 0.687 , respectively) (Fig. 1C).

To explore the survival benefit from adjuvant chemotherapy by joint markers, we categorized patients into the follow-

Table 4. Disease-Free Survival Analyzed by a Multivariate Cox Proportional-Hazards Model

\begin{tabular}{|c|c|c|}
\hline & HR (95\% CI) & $p$ value \\
\hline Age & $1.015(1.003-1.027)$ & 0.013 \\
\hline Treatment & & $<0.001$ \\
\hline Surgery only & Reference & \\
\hline Surgery+CTx & $0.621(0.475-0.811)$ & \\
\hline pTNM stage ${ }^{*}$ & & $<0.001$ \\
\hline$\|$ & Reference & \\
\hline III & 2.160 (1.621-2.879) & \\
\hline Lauren classification & & 0.024 \\
\hline Intestinal & Reference & \\
\hline Non-intestinal & $1.421(1.048-1.927)$ & \\
\hline MSI status & & 0.015 \\
\hline MSS/MSI-L & Reference & \\
\hline MSI-H & $0.363(0.161-0.820)$ & \\
\hline SPC prognosis & & 0.003 \\
\hline Low-risk & Reference & \\
\hline Intermediate-risk & $1.879(1.101-3.205)$ & 0.021 \\
\hline High-risk & $2.399(1.415-4.067)$ & 0.001 \\
\hline
\end{tabular}

$\mathrm{HR}$, hazard ratio; $\mathrm{Cl}$, confidence interval; CTx, adjuvant chemotherapy (capecitabine plus oxaliplatin); SPC, single patient classifier; MSI, microsatellite instability; MSS, microsatellite stable; MSI-L, microsatellite instabilitylow; MSI-H, microsatellite instability-high.

${ }^{*}$ According to American Joint Committee on Cancer 6th edition. ing: 1) responder/MSI-H, non-responder/MSI-H, responder/ MSS/MSI-L, and non-responder/MSS/MSI-L for joint analysis of SPC and MSI and 2) responder/EBV-positive, non-responder/EBV-positive, responder/EBV-negative, and non-responder/EBV-negative for joint analysis of SPC and EBV.

In both non-responder/MSI-H and responder/MSI-H populations, no prognostic difference was observed between surgery alone and surgery plus chemotherapy ( $p=0.630$ and 0.317 , respectively). In contrast, in MSS/MSI-L patients, a significant survival benefit from adjuvant chemotherapy was seen in responder group of SPC-prediction ( $p=0.001)$ (Fig. $2 \mathrm{~A}$ ), but not in non-responder group ( $p=0.168$ ) (Fig. 2B).

In case of joint SPC/EBV, for responder/EBV-positive patients, survival benefit from adjuvant chemotherapy was not analyzed since all of the three patients underwent surgery alone. In the remaining population, the trend of survival benefit in joint SPC/EBV was similar to that for joint SPC/MSI. In non-responder/EBV-positive patients, no survival benefit from adjuvant chemotherapy was observed ( $p=0.491 ; \mathrm{n}=18$ and 20 for surgery alone and adjuvant chemotherapy group, respectively). For EBV-negative patients, responder group of SPC-prediction gained a significant survival benefit from adjuvant chemotherapy ( $p=0.006$ ) (Fig. 2C), whereas non-responder group did not ( $p=0.587$ ) (Fig. 2D).

\section{DISCUSSION}

We examined the combinatory effect of clinically relevant subtypes, SPC, MSI, and EBV, in the prediction of prognosis and chemotherapy response after surgery using a cohort of 586 patients enrolled in CLASSIC trial, a randomized controlled study of capecitabine plus oxaliplatin-based adjuvant chemotherapy for stage II/III gastric cancer. The three markers used in this study, SPC, MSI, and EBV, were based on different biological, pathological, or etiological grounds. SPC classified patients by immune, stem-like, and epithelial characteristics from the gene expression profile of tumor tissues, while MSI-H and EBV-positive tumors were associated with genetic hypermutability due to impaired DNA mismatch repair and exogeneous viral infection, respectively. Thus, we expected that our joint marker analysis would discover new findings clinically meaningful to patient selection. It was also considered that the use of these markers or some of their combinations would be comparatively feasible to be introduced into practice.

In the analyses evaluating the combinatory prognostic effects of SPC, MSI, and EBV, joint SPC/MSI and SPC/EBV showed their respective clinical implications. 1) SPC and MSI were independent prognostic factors. Although i) MSI-H tumors have been known for their activated immune response and ii) SPC and MSI have a positive association, the proportion of MSI-H was slightly higher in low-risk group than in 

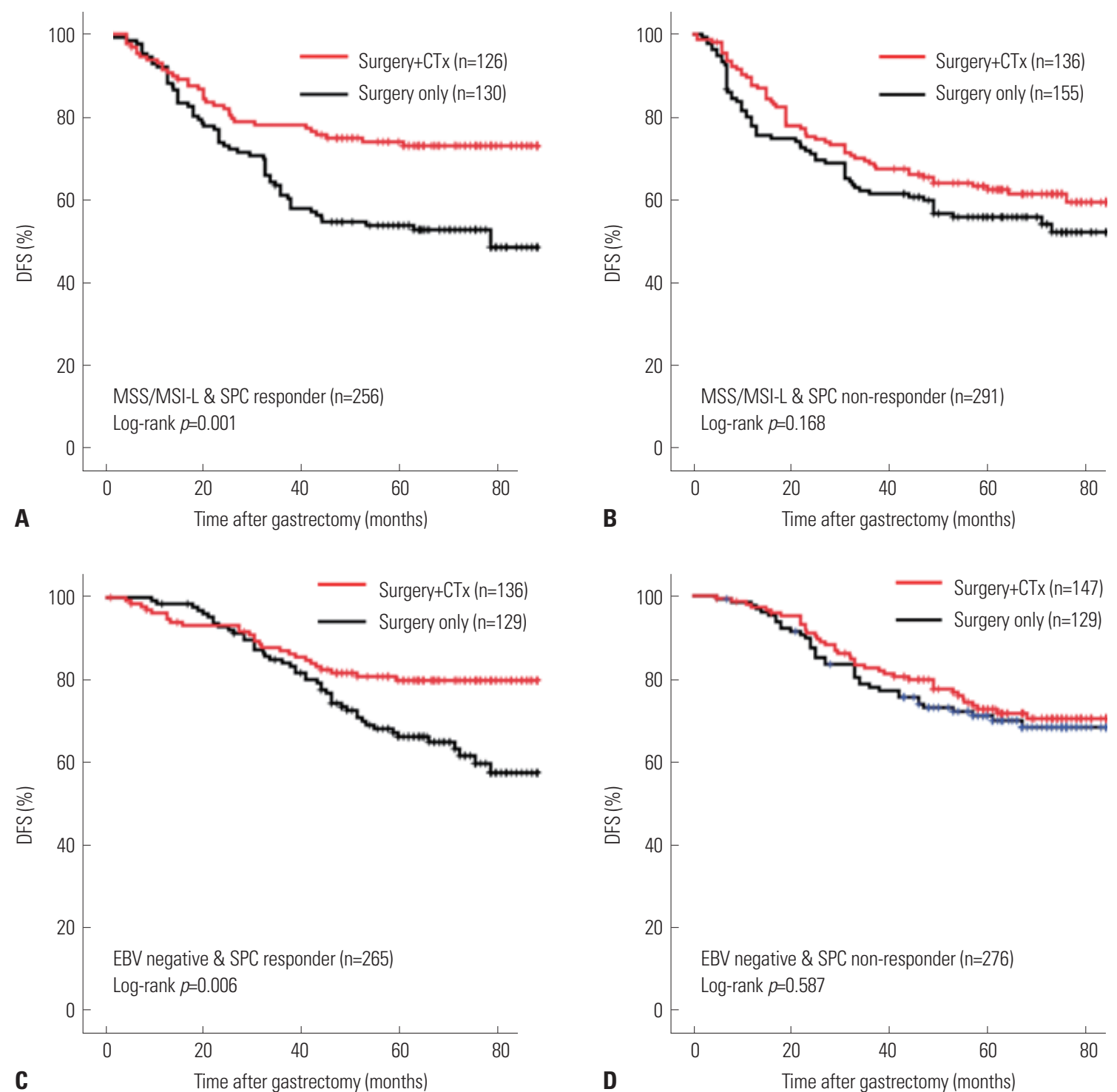

Fig. 2. Kaplan-Meier and log-rank comparisons of DFS in patients with stage II/III gastric cancer following treatment in subgroups of (A) MSS/MSI-L and SPC responder group, (B) MSS/MSI-L and SPC non-responder group, (C) EBV-negative and SPC responder group, and (D) EBV-negative and SPC nonresponder group. DFS, disease-free survival; CTx, adjuvant chemotherapy (capecitabine plus oxaliplatin); MSS, microsatellite stable; MSI-L, microsatellite instability-low; SPC, single patient classifier; EBV, Epstein-Barr virus.

other groups ( $p<0.001$; chi square test) and low-risk/MSS/ MSI-L patients had as good a prognosis as MSI-H patients ( $p=0.001$; log-rank test), indicating that aside from MSI status, SPC identifies patients whose long-term survival is anticipated. 2) EBV was not a significant prognostic factor in this cohort. SPC/EBV, however, showed the most dramatic stratification between low-risk and high-risk patients in EBV-positive group. As low-risk and high-risk groups in SPC scheme were associated with immune and stem-like characteristics, respectively, the result implies that not all EBV-positive patients might have the same immune response, supporting the existence of molecular heterogeneity in EBV-associated gastric cancers. $^{21,22}$

SPC, MSI, and EBV statuses identified two groups of patients in the present CLASSIC cohort: those who gained sur- vival benefit from adjuvant chemotherapy and those who did not. The combination of SPC/MSI or SPC/EBV led to a more elaborate selection of these patient groups. 1) It has generally been accepted that MSI-H patients do not benefit from chemotherapy, whereas MSS/MSI-L patients do. ${ }^{5,12,13}$ The combinatory SPC and MSI statuses further classified patients in MSS/MSI-L population who did not benefit from chemotherapy. 2) SPC/EBV worked in a manner similar to that of SPC/ MSI. EBV-positive patients did not gain chemotherapy benefits, while EBV-negative patients did. Joint SPC/EBV further selected patients from EBV-negative population who did not gain chemotherapy benefits. The combinatory use of SPC, MSI, and EBV might guide clinical decisions with regards to chemotherapeutic treatment.

Currently, accumulating studies have reported that patients 
with MSI-H and EBV-positive gastric cancer dramatically responded to immune checkpoint inhibitors. ${ }^{23}$ As defined by the immune-related characteristics, low-risk/non-responder SPC subtype could be another companion diagnostic candidate for immune therapy. Otherwise, the combinatory use of SPC, MSI, and EBV may yield meaningful knowledge on the immunological landscape of gastric cancer, leading to a better selection of patients for immune therapy and improvement in response rates.

This study had some limitations. First, although the distribution of clinical variables of the study cohort $(n=586)$ was similar to that of the original CLASSIC trial cohort $(n=1035)$, the possibility of selection bias cannot be overlooked. Second, due to a limited number of cases and events, type II error needs to be considered, especially in subgroup analysis. Although the present results were from a randomized controlled trial (RCT), additional validation with qualified specimens should be conducted to reach a substantial consensus of the clinical role of these markers in patients with gastric cancer. Using specimens from other RCTs, such as MAGIC, ${ }^{12,24}$ ARTIST, ${ }^{25}$ or FLOT ${ }^{26}$ trials, would be the most reliable and practical option.

In conclusion, SPC, MSI, and EBV positivity could be used in a combinatory manner to predict the prognosis and responsiveness of adjuvant chemotherapy for stage II/III gastric cancer.

\section{ACKNOWLEDGEMENTS}

This research was supported by a grant from the National R\&D Program for Cancer Control, Ministry of Health and Welfare, Republic of Korea (1020390, 1320360).

\section{AUTHOR CONTRIBUTIONS}

Conceptualization: Chul Kyu Roh, Yoon Young Choi, Jae-Ho Cheong. Data curation: Chul Kyu Roh, Yoon Young Choi, Seohee Choi, Won Jun Seo, Minah Cho, Taeil Son, Hyoung-Il Kim, Woo Jin Hyung, Sung Hoon Noh, Jae-Ho Cheong. Formal analysis: Chul Kyu Roh, Yoon Young Choi. Funding acquisition: Sung Hoon Noh, Jae-Ho Cheong. Investigation: Chul Kyu Roh, Yoon Young Choi, Seohee Choi, Won Jun Seo, Minah Cho, Taeil Son, Hyoung-Il Kim, Woo Jin Hyung, Sung Hoon Noh, Jae-Ho Cheong. Methodology: Yoon Young Choi, Eunji Jang, Hyeseon Kim. Project administration: Yong-Min Huh, Jae-Ho Cheong. Resources: Chul Kyu Roh, Yoon Young Choi, Seohee Choi, Won Jun Seo, Minah Cho, Taeil Son, Hyoung-Il Kim, Woo Jin Hyung, Sung Hoon Noh, Jae-Ho Cheong. Software: Chul Kyu Roh, Yoon Young Choi, Eunji Jang, Hyeseon Kim. Supervision: Jae-Ho Cheong. Validation: Chul Kyu Roh, Yoon Young Choi, Seohee Choi, Won Jun Seo, Minah Cho, Taeil Son, Hyoung-Il Kim, Woo Jin Hyung, Sung Hoon Noh, Jae-Ho Cheong. Visualization: Chul Kyu Roh, Yoon Young Choi. Writing_original draft: Chul Kyu Roh, Yoon Young Choi, Eunji Jang. Writing—review \& editing: Jae-Ho Cheong.

\section{ORCID iDs}

Yoon Young Choi https://orcid.org/0000-0002-2179-7851 Seohee Choi https://orcid.org/0000-0002-9384-8646 Won Jun Seo https://orcid.org/0000-0003-3447-7790 Minah Cho https://orcid.org/0000-0003-3011-5813 Eunji Jang https://orcid.org/0000-0002-5023-5006 Taeil Son https://orcid.org/0000-0002-0327-5224 Hyoung-Il Kim https://orcid.org/0000-0002-6134-4523 Hyeseon Kim https://orcid.org/0000-0002-4964-1373 Woo Jin Hyung https://orcid.org/0000-0002-8593-9214 Yong-Min Huh https://orcid.org/0000-0002-9831-4475 Sung Hoon Noh $\quad$ https://orcid.org/0000-0003-4386-6886 Jae-Ho Cheong https://orcid.org/0000-0002-1703-1781

\section{REFERENCES}

1. Choi YY, Cheong JH. Beyond precision surgery: molecularly motivated precision care for gastric cancer. Eur J Surg Oncol 2017;43: 856-64.

2. Choi YY, Noh SH, Cheong JH. Evolution of gastric cancer treatment: from the golden age of surgery to an era of precision medicine. Yonsei Med J 2015;56:1177-85.

3. Choi YY, Noh SH, Cheong JH. Molecular dimensions of gastric cancer: translational and clinical perspectives. J Pathol Transl Med 2016;50:1-9.

4. Bang YJ, Van Cutsem E, Feyereislova A, Chung HC, Shen L, Sawaki A, et al. Trastuzumab in combination with chemotherapy versus chemotherapy alone for treatment of HER2-positive advanced gastric or gastro-oesophageal junction cancer (ToGA): a phase 3, open-label, randomised controlled trial. Lancet 2010; 376:687-97.

5. Ribic CM, Sargent DJ, Moore MJ, Thibodeau SN, French AJ, Goldberg RM, et al. Tumor microsatellite-instability status as a predictor of benefit from fluorouracil-based adjuvant chemotherapy for colon cancer. N Engl J Med 2003;349:247-57.

6. Paik S, Shak S, Tang G, Kim C, Baker J, Cronin M, et al. A multigene assay to predict recurrence of tamoxifen-treated, node-negative breast cancer. N Engl J Med 2004;351:2817-26.

7. Cancer Genome Atlas Research Network. Comprehensive molecular characterization of gastric adenocarcinoma. Nature 2014;513:202-9.

8. Tan IB, Ivanova T, Lim KH, Ong CW, Deng N, Lee J, et al. Intrinsic subtypes of gastric cancer, based on gene expression pattern, predict survival and respond differently to chemotherapy. Gastroenterology 2011;141:476-85.

9. Cristescu R, Lee J, Nebozhyn M, Kim KM, Ting JC, Wong SS, et al. Molecular analysis of gastric cancer identifies subtypes associated with distinct clinical outcomes. Nat Med 2015;21:449-56.

10. Choi YY, Bae JM, An JY, Kwon IG, Cho I, Shin HB, et al. Is microsatellite instability a prognostic marker in gastric cancer? A systematic review with meta-analysis. J Surg Oncol 2014;110:129-35.

11. Kim SY, Choi YY, An JY, Shin HB, Jo A, Choi H, et al. The benefit of microsatellite instability is attenuated by chemotherapy in stage II and stage III gastric cancer: results from a large cohort with subgroup analyses. Int J Cancer 2015;137:819-25.

12. Smyth EC, Wotherspoon A, Peckitt C, Gonzalez D, Hulkki-Wilson S, Eltahir Z, et al. Mismatch repair deficiency, microsatellite instability, and survival: an exploratory analysis of the Medical Research Council Adjuvant Gastric Infusional Chemotherapy (MAGIC) Trial. JAMA Oncol 2017;3:1197-203.

13. Choi YY, Kim H, Shin SJ, Kim HY, Lee J, Yang HK, et al. Microsatellite instability and programmed cell death-ligand 1 expression in stage II/III gastric cancer: post hoc analysis of the CLASSIC ran- 
domized controlled study. Ann Surg 2018 May 1 [Epub]. Available at: https://doi.org/10.1097/SLA.0000000000002803.

14. Choi YY, Cheong JH. To treat, or not to treat, that is the question: biomarker-guided adjuvant chemotherapy for stage II and III gastric cancer. Ann Surg 2018 Nov 22 [Epub]. Available at: https:// doi.org/10.1097/SLA.0000000000003102.

15. Cheong JH, Yang HK, Kim H, Kim WH, Kim YW, Kook MC, et al. Predictive test for chemotherapy response in resectable gastric cancer: a multi-cohort, retrospective analysis. Lancet Oncol 2018; 19:629-38.

16. Gambardella V, Cervantes A. Precision medicine in the adjuvant treatment of gastric cancer. Lancet Oncol 2018;19:583-4.

17. Bang YJ, Kim YW, Yang HK, Chung HC, Park YK, Lee KH, et al. Adjuvant capecitabine and oxaliplatin for gastric cancer after D2 gastrectomy (CLASSIC): a phase 3 open-label, randomised controlled trial. Lancet 2012;379:315-21.

18. Noh SH, Park SR, Yang HK, Chung HC, Chung IJ, Kim SW, et al. Adjuvant capecitabine plus oxaliplatin for gastric cancer after D2 gastrectomy (CLASSIC): 5-year follow-up of an open-label, randomised phase 3 trial. Lancet Oncol 2014;15:1389-96.

19. Patil DT, Bronner MP, Portier BP, Fraser CR, Plesec TP, Liu X. A five-marker panel in a multiplex PCR accurately detects microsatellite instability-high colorectal tumors without control DNA. Diagn Mol Pathol 2012;21:127-33.

20. Goel A, Nagasaka T, Hamelin R, Boland CR. An optimized pentaplex PCR for detecting DNA mismatch repair-deficient colorectal cancers. PLoS One 2010;5:e9393.

21. Kim HS, Shin SJ, Beom SH, Jung M, Choi YY, Son T, et al. Compre- hensive expression profiles of gastric cancer molecular subtypes by immunohistochemistry: implications for individualized therapy. Oncotarget 2016;7:44608-20.

22. Park JH, Kim EK, Kim YH, Kim JH, Bae YS, Lee YC, et al. EpsteinBarr virus positivity, not mismatch repair-deficiency, is a favorable risk factor for lymph node metastasis in submucosa-invasive early gastric cancer. Gastric Cancer 2016;19:1041-51.

23. Kim ST, Cristescu R, Bass AJ, Kim KM, Odegaard JI, Kim K, et al. Comprehensive molecular characterization of clinical responses to PD-1 inhibition in metastatic gastric cancer. Nat Med 2018;24: 1449-58.

24. Cunningham D, Allum WH, Stenning SP, Thompson JN, Van de Velde CJ, Nicolson M, et al. Perioperative chemotherapy versus surgery alone for resectable gastroesophageal cancer. N Engl J Med 2006;355:11-20.

25. Park SH, Sohn TS, Lee J, Lim DH, Hong ME, Kim KM, et al. Phase III trial to compare adjuvant chemotherapy with capecitabine and cisplatin versus concurrent chemoradiotherapy in gastric cancer: final report of the Adjuvant Chemoradiotherapy in Stomach Tumors Trial, including survival and subset analyses. J Clin Oncol 2015;33:3130-6.

26. Al-Batran SE, Pauligk C, Homann N, Schmalenberg H, Kopp HG, Haag GM, et al. LBA-008: Docetaxel, oxaliplatin, and fluorouracil/ leucovorin (FLOT) versus epirubicin, cisplatin, and fluorouracil or capecitabine (ECF/ECX) as perioperative treatment of resectable gastric or gastro-esophageal junction adenocarcinoma: the multicenter, randomized phase 3 FLOT4 trial (German Gastric Group at AIO). Ann Oncol 2017;28(suppl_3):mdx302.007. 